\title{
Numerical Manifold Method with Endochronic Theory for Elastoplasticity Analysis
}

\author{
Wei Zeng, Junjie Li, and Fei Kang \\ Faculty of Infrastructure Engineering, Dalian University of Technology, Dalian, Liaoning 116024, China \\ Correspondence should be addressed to Wei Zeng; zengw12345678@163.com
}

Received 29 November 2013; Accepted 7 January 2014; Published 27 February 2014

Academic Editor: Ting-Hua Yi

Copyright (C) 2014 Wei Zeng et al. This is an open access article distributed under the Creative Commons Attribution License, which permits unrestricted use, distribution, and reproduction in any medium, provided the original work is properly cited.

\begin{abstract}
Numerical manifold method (NMM) was originally developed based on linear elastic constitutive model. For many problems it is difficult to obtain accurate results without elastoplasticity analysis, and an elastoplasticity version of NMM is needed. In this paper, the incremental endochronic theory is extended into NMM analysis and an endochronic NMM algorithm is proposed for elastoplasticity analysis. It is well known that endochronic theory is one of the widely used elastoplasticity theories which can deal with elastoplasticity problems without a yield surface and loading or unloading judgments. Numerical tests show that the proposed algorithm of endochronic NMM possesses a good accuracy. The proposed algorithm is also applied to analyze a crack problem and a soft clay foundation under traffic loading problem. Results demonstrate the convenience of the endochronic NMM in analyzing elastoplasticity discontinuous problems.
\end{abstract}

\section{Introductions}

The numerical manifold method (NMM) was proposed by Shi $[1,2]$. It is a numerical analysis method for integrating the discontinuous deformation analysis (DDA) and the finite element method (FEM). The NMM is based on the finite cover approximation theory and approximate displacement by solving the equation on covers. Comparing with FEM, the NMM has the following advantages [3]: (1) the geometry of a model can be discontinuous; (2) it can solve problems with large deformation; (3) for quasistatic problems, the condition of equilibrium is automatically satisfied; (4) there is no need for Gauss integration points by using the simplex integration. Since the original version of NMM proposed, various developments and applications have been achieved during these years [4]. Ma et al. [5] and Gao and Cheng [6] applied NMM to analyze fracture problems. Zhang et al. [7] extended it to predict the growth of complex cracks. Terada and Kurumatani [8] proposed a version of NMM called the finite cover method. Li and Cheng [9] developed meshless NMM based on unit partition. Meshless NMM was extended into crack problems by Li and Cheng [10]. Zhang and Zhou [11] applied NMM to analyze the saturated porous media. Gao and Cheng [12] introduced the complex variable into NMM.
Already developed NMM algorithms mainly adopted a linear elastic constitutive model. However, it is difficult to obtain accurate results without elastoplasticity analysis for many problems, and an elastoplasticity version of NMM is needed.

Endochronic theory is an elastoplasticity constitutive theory derived from thermo concepts by Valanis $[13,14]$ in 1971. It is widely used in metal, sand, concrete, and so on [15, 16]. Comparing with other plasticity theories, endochronic theory has neither conception of a yield surface nor the specification of the loading or unloading rules and can take the effects of the strain history into account by using intrinsic time. Unfortunately, endochronic FEM is not good at dealing with discontinuous problems and mass movement problems which NMM is expert in.

In this paper, we introduce the endochronic theory into NMM and apply the proposed algorithm to analyze a crack problem and a soft clay foundation under traffic loading problem. This paper is organized as follows. In Section 2, the basic conception of NMM and the algorithm of endochronic NMM are described. In Section 3, the accuracy of endochronic NMM is verified by numerical tests. In Section 4 , numerical examples are taken to prove the availability of the algorithm. In Section 5, conclusions are given. 


\section{Endochronic NMM}

2.1. The Basic Conceptions of NMM. There are three main components that form the heart of NMM: block kinematics, simplex integration method, and finite covering systems. The block kinematics theory is developed from DDA which can deal with the problems of contact, large deformation, and mass movement. The integration of a function in NMM is evaluated by simplex integration method. This integration method can be implemented on any shape without Gauss integration points. In NMM, there are two sets of covers. One is called physical cover (PC) which contains all the physical features such as the internal face, the domain which needs to be simulated, and the geometries on which the boundary conditions are loaded. Another kind of cover is called mathematical cover (MC) which must cover the physical cover. For example in Figure $1(\mathrm{a}), \Omega_{1}$ and $\Omega_{2}$ are the physical domains for analysis and Figure 1(b) shows the mathematical domain. In Figure $1(\mathrm{c})$, the $\mathrm{MC}_{1}$ and $\mathrm{MC}_{2}$ are the mathematical covers and Figure $1(\mathrm{~d})$ shows the physical covers $\left(\mathrm{PC}_{1-4}\right)$. The manifold elements (MEs) are the intersection of mathematical covers and physical covers.

NMM is a numerical method based on the principle of minimum total potential energy. The weak form of governing equation is defined as

$$
\int_{\Omega} \delta \varepsilon^{T} \Delta \sigma d V+\int_{\Omega} \delta u^{T} \rho \ddot{u} d V=\int_{\Omega} \delta u^{T} \Delta b d V+\int_{\Gamma_{t}} \delta u^{T} \Delta \bar{t} d \Gamma,
$$

where $\sigma$ is the Cauchy stress tensor, $\varepsilon$ is the small strain tensor, $u$ is the displacement vector, $\ddot{u}$ is the acceleration vector, $\rho$ is the density of the material, $b$ is the body force vector, and $\bar{t}$ is the traction vector applied to boundary $\Gamma_{t}$.

2.2. The Incremental Constitutive Equations. Constitutive equations of endochronic theory for homogeneous and isotropic materials could be described as follows:

$$
\begin{gathered}
\sigma_{\mathrm{dev}}=2 \int_{0}^{z} \rho\left(z-z^{\prime}\right) \frac{\partial \varepsilon_{\mathrm{dev}}^{P}}{\partial z^{\prime}} d z^{\prime} \\
d \varepsilon_{\mathrm{dev}}^{p}=d \varepsilon_{\mathrm{dev}}-\frac{d \sigma_{\mathrm{dev}}}{2 \mu}
\end{gathered}
$$

where $\mu$ is the elastic shear modulus, $\sigma_{\mathrm{dev}}$ is the deviatoric stress, and $\varepsilon_{\mathrm{dev}}^{p}$ is the deviatoric plastic strain. In (2), $Z$ is the intrinsic time measure which can be defined as follows:

$$
\begin{gathered}
d z=\frac{d \xi}{f(\xi)} \\
d \xi^{2}=d \varepsilon_{\mathrm{dev}}^{p}: d \varepsilon_{\mathrm{dev}}^{p}
\end{gathered}
$$

where $f(\xi)$ is called scale function, and it decides the behavior of material softening or hardening. By this function, the contribution of the plastic strain history can be taken into account. $\rho(z)$ is a kernel function and the $m$-term Dirichlet series are introduced into this function by Lee [17], which can be written as

$$
\rho(z)=\sum_{i=1}^{n} C_{i} e^{-\alpha_{i} z}
$$

with the requirement that $C_{i}$ and $\alpha_{i}$ are positive for all $i$ and

$$
\sum_{i=1}^{\infty} C_{i}=\infty, \quad \sum_{i=1}^{\infty} \frac{C_{i}}{\alpha_{i}}<\infty
$$

where $C$ and $\beta$ are material parameters.

The incremental form of endochronic NMM which is used in the numerical simulation can be deduced from the integral constitutive equations mentioned above. We assume that the variables at step $n-1$ are known, and the increment of deviatoric stress in step $n$ can be described as

$$
\Delta \sigma_{\mathrm{dev}}\left({ }^{n} z\right)=\sigma_{\mathrm{dev}}\left({ }^{n} z\right)-\sigma_{\mathrm{dev}}\left({ }^{n-1} z\right)
$$

Substituting (2) and (5) to (7),

$$
\begin{aligned}
\Delta \sigma_{\mathrm{dev}}\left({ }^{n} z\right)= & 2 \frac{{ }^{n} \Delta \varepsilon_{\mathrm{dev}}^{P}}{{ }^{n} \Delta z} \sum_{r=1}^{m} \frac{C_{r}}{\alpha_{r}}\left(1-e^{-\alpha_{r}{ }^{n} \Delta z}\right) \\
& +\sum_{r=1}^{m} \sigma_{\mathrm{dev}}^{r}\left({ }^{n-1} z\right)\left(e^{-\alpha_{r}{ }^{n} \Delta z}-1\right) .
\end{aligned}
$$

It can be easy derived that

$$
\begin{aligned}
\Delta \sigma_{\mathrm{dev}}\left({ }^{n} z\right)= & 2 \frac{{ }^{n} \Delta \varepsilon_{\mathrm{dev}}}{{ }^{n} \Delta z^{n} M} \sum_{r=1}^{m} \frac{C_{r}}{\alpha_{r}}\left(1-e^{-\alpha_{r}{ }^{n} \Delta z}\right) \\
& +\frac{1}{{ }^{n} M} \sum_{r=1}^{m} \sigma_{\mathrm{dev}}^{r}\left({ }^{n-1} z\right)\left(e^{-\alpha_{r}{ }^{n} \Delta z}-1\right) \\
{ }^{n} M= & +\frac{1}{{ }^{n} \Delta z \mu} \sum_{r=1}^{m} \frac{C_{r}}{\alpha_{r}}\left(1-e^{-\alpha_{r}{ }^{n} \Delta z}\right) .
\end{aligned}
$$

We assume that the material is plastic incompressibility and the hydrostatic response can be expressed as

$$
\Delta \sigma_{h}=3 K \Delta \varepsilon_{\mathrm{vol}}
$$

where $K$ is the elastic bulk modulus. The total stress $\Delta \sigma$ is that

$$
\Delta \sigma=\Delta \sigma_{\mathrm{dev}}+\Delta \sigma_{h} I
$$

Substituting (9) and (11) to (12), the incremental stress vector can be represented as follows:

$$
\begin{aligned}
{ }^{n} \Delta \sigma= & 3 K^{n} \Delta \varepsilon_{\mathrm{vol}} I+2 \frac{{ }^{n} \Delta \varepsilon_{\mathrm{dev}}}{{ }^{n} \Delta z^{n} M} \sum_{r=1}^{m} \frac{C_{r}}{\alpha_{r}}\left(1-e^{\left.-\alpha_{r}{ }^{n} \Delta z\right)}\right. \\
& +\frac{1}{{ }^{n} M} \sum_{r=1}^{m} \sigma_{\mathrm{dev}}^{r}\left({ }^{n-1} z\right)\left(e^{-\alpha_{r}{ }^{n} \Delta z}-1\right) .
\end{aligned}
$$




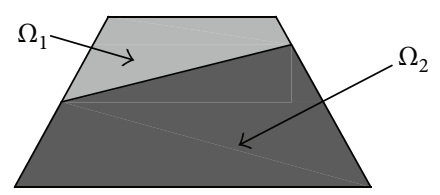

(a) Physical domain

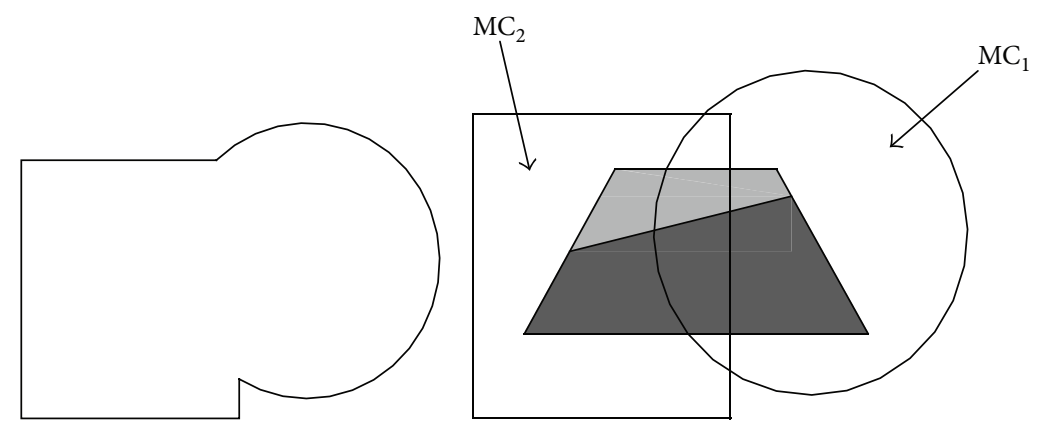

(b) Mathematical domain

(c) Mathematical covers

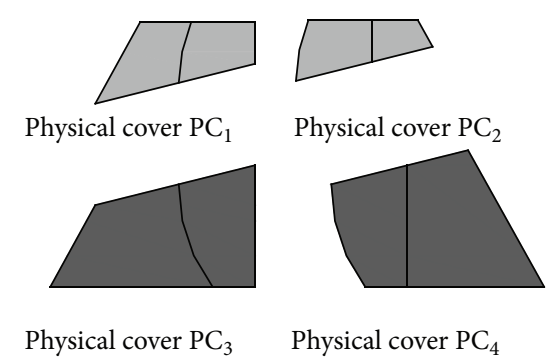

(d) Physical covers

Figure 1: Mathematical covers and physical covers.

2.3. Discrete Equations for Endochronic NMM. In a manifold element, the relationship of the displacement vector and the strain tensor in (1) can be written as

$$
\begin{gathered}
u=N d, \\
\varepsilon=L N d=B d,
\end{gathered}
$$

where $N$ is the shape function, $L$ is the differential operator matrix, and $d$ is the unknown vector. From the constitutive equations, the stress tensor can be written as follows:

$$
\Delta \sigma=D^{\mathrm{ep}} \Delta \varepsilon+f^{\prime},
$$

where the elastoplasticity matrix $D^{\mathrm{ep}}$ and vector $f^{\prime}$ are written as follows:

$$
\begin{gathered}
D^{\text {ep }}=\left[\begin{array}{cccccc}
A_{1} & A_{2} & A_{2} & 0 & 0 & 0 \\
A_{2} & A_{1} & A_{2} & 0 & 0 & 0 \\
A_{2} & A_{2} & A_{1} & 0 & 0 & 0 \\
0 & 0 & 0 & A_{3} & 0 & 0 \\
0 & 0 & 0 & 0 & A_{3} & 0 \\
0 & 0 & 0 & 0 & 0 & A_{3}
\end{array}\right], \\
A_{1}=K+\frac{4 \sum_{r=1}^{m}\left(C_{r} / \alpha_{r}\right)\left(1-e^{\left.-\alpha_{r}{ }^{n} \Delta z\right)}\right.}{3^{n} \Delta z^{n} M}, \\
A_{2}=K-\frac{2 \sum_{r=1}^{m}\left(C_{r} / \alpha_{r}\right)\left(1-e^{\left.-\alpha_{r}{ }^{n} \Delta z\right)}\right.}{3^{n} \Delta z^{n} M}
\end{gathered}
$$

$$
\begin{gathered}
A_{3}=\frac{\sum_{r=1}^{m}\left(C_{r} / \alpha_{r}\right)\left(1-e^{-\alpha_{r}{ }^{n} \Delta z}\right)}{{ }^{n} \Delta z^{n} M}, \\
f^{\prime}=\frac{1}{{ }^{n} M} \sum_{r=1}^{m} \sigma_{\operatorname{dev}}^{r}\left({ }^{n-1} z\right)\left(e^{-\alpha_{r}{ }^{n} \Delta z}-1\right) .
\end{gathered}
$$

Substituting (14) and (15) to (1), we finally obtain the discrete equations for endochronic NMM analysis:

$$
K \Delta d+M \Delta \ddot{d}=\Delta F,
$$

where the stiffness matrix $K$, mass matrix $M$, and the incremental load $\Delta F$ can be represented as:

$$
\begin{gathered}
K=\int_{\Omega} B^{T} D^{\mathrm{ep}} B d \Omega \\
M=\int_{\Omega} \rho N^{T} N d V \\
\Delta F=\int_{\Omega} N^{T} \Delta b d \Omega+\int_{\Gamma} N^{T} \Delta \bar{t} d \Gamma+\int_{\Omega} B^{T} f^{\prime} d \Omega .
\end{gathered}
$$

To consider the dynamic problems, the total time is divided into limited number of periods. By using the Newmark scheme, the acceleration vector can be written as

$$
\begin{gathered}
d_{n+1}=d_{n}+\Delta t_{n} \dot{d}_{n}+\frac{\Delta t_{n}^{2}}{2}\left((1-\beta) \ddot{d}_{n}+\beta \ddot{d}_{n+1}\right) \\
\dot{d}_{n+1}=\dot{d}_{n}+\Delta t_{n}\left((1-\gamma) \ddot{d}_{n}+\gamma \ddot{d}_{n+1}\right)
\end{gathered}
$$


TABLE 1: Description of the algorithm.

\begin{tabular}{|c|c|}
\hline Step & Description \\
\hline 1 & $\begin{array}{l}\text { Assume the state at step } n-1 \text { is known, and step } n \text { is } \\
\text { the current step }\end{array}$ \\
\hline 2 & $\begin{array}{l}\text { The prediction solution is obtained by calculation with } \\
\text { elastic constitutive. Update the incremental stress } \\
\text { matrix }\end{array}$ \\
\hline 4 & Initialize $\Delta \xi$ \\
\hline 5 & Use the Newton-Raphson technique to calculate ${ }^{n} \Delta z$ by \\
\hline 6 & Substitute ${ }^{n} \Delta z$ to (15) and calculate $D^{\mathrm{ep}}$ and $f^{\prime}$ \\
\hline 7 & $\begin{array}{l}\text { Solve (22) for } \Delta d \text {. Update the global displacements } \Delta u \\
\text { and the global incremental strain matrix } \Delta \varepsilon\end{array}$ \\
\hline 8 & Go to the next step. \\
\hline
\end{tabular}

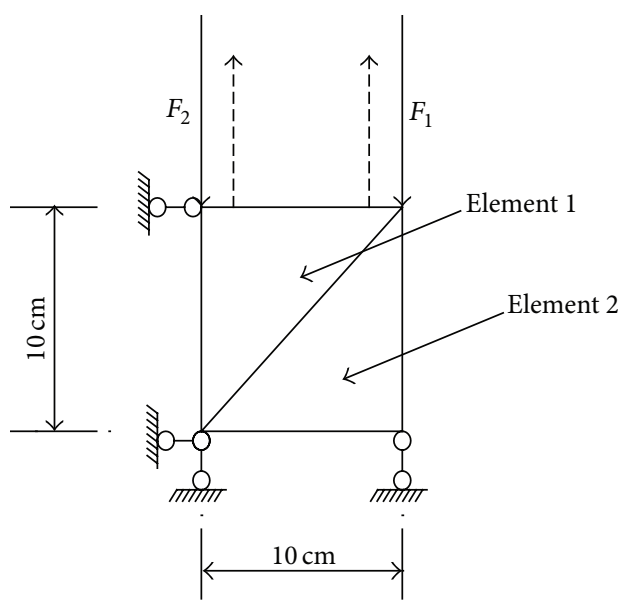

Figure 2: Geometry and mathematic mesh for cyclic loading.

and then

$$
\ddot{d}_{n+1}=\frac{2}{\Delta t^{2} \beta}\left(\Delta d-\Delta t \dot{d}_{n}-\frac{\Delta t^{2}}{2}(1-\beta) \ddot{d}_{n}\right) .
$$

Here we defined $\beta$ and $\gamma$ equal to 1 . The acceleration vector is transformed as

$$
\ddot{d}_{n+1}=\frac{2}{\Delta t^{2}}\left(\Delta d-\Delta t \dot{d}_{n}\right)
$$

The discrete equations for dynamic problem derived from (17) and (21) can be written as

$$
\bar{K} \Delta d=\Delta \bar{F},
$$

where

$$
\bar{K}=K+\frac{2}{\Delta t^{2}} M, \quad \Delta \bar{F}=\Delta F+\frac{2 V_{n}}{\Delta t} M .
$$

The details of the algorithm used in the program are described in Table 1.

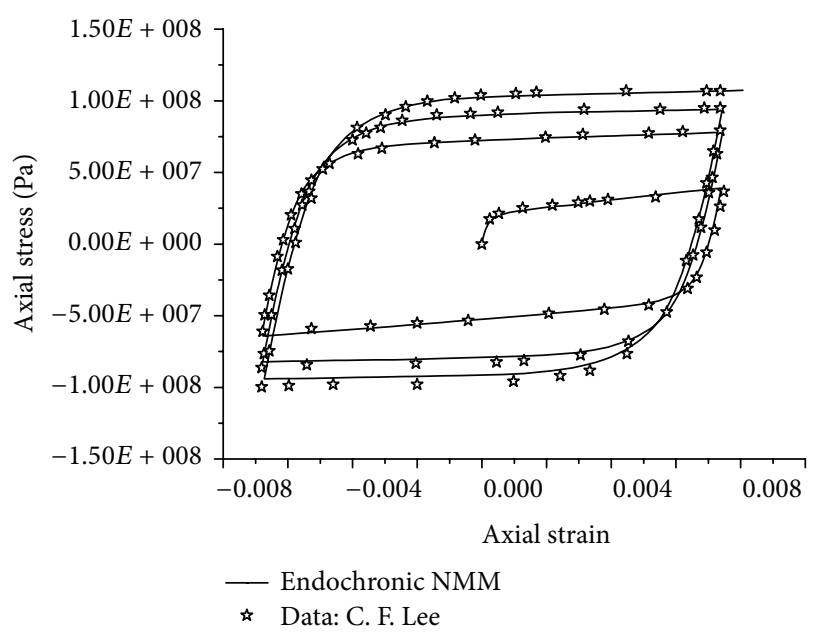

Figure 3: The axial stress versus strain relation of element 1.

\section{Performance of Endochronic NMM}

3.1. Test A: Behaviors under Cyclic Loading. This example is taken from Lee [17]. He applied the endochronic theory to an oxygen-free high-conductivity copper plate under cyclic loading with FEM and the results coincide with the experimental data [18] very well. In this section, a number of experiments are performed to test the accuracy of the endochronic NMM. The geometry and mathematic mesh of the problem are shown in Figure 2. $F_{1}$ and $F_{2}$ are the forces which are applied on the node of the model and the values of $F_{1}$ and $F_{2}$ are adjusted to ensure that the axial stress is equal to the date from literature [17]. In order to overlook the effect of mathematic covers, we let the mathematic domain have the same area as physical domain. The material parameters, which are provided by Lee, are listed in Table 2 and the material functions: $\rho(z)$ and $f(\xi)$ are defined as follows:

$$
\begin{gathered}
\rho(z)=\sum_{i=1}^{7} C_{i} e^{-\alpha_{i} z}, \\
f(\xi)= \begin{cases}1-0.84 e^{-16.4 \xi}, & \zeta \leq 0.035, \\
1-0.637 e^{-8.48 \xi}, & \zeta>0.035 .\end{cases}
\end{gathered}
$$

Figure 3 shows that the results from endochronic NMM are identical to the data from Lee [17]. This example can demonstrate that when the mathematic mesh fits with the physical covers, the endochronic NMM will produce exactly the same result as endochronic FEM.

3.2. Test B: The Influence of Mathematical Covers. In FEM, the mesh is identical with the geometric figure, but in NMM the mathematic mesh is arbitrary and the integral form is different from FEM. In this section, as shown in Figure 4, we study the influences of the mathematical mesh by changing the length and position of mathematical cover. We apply a single force on the highest point of the model. Figure 5 is the cyclic loading diagram. The parameters of the endochronic model are shown in Table 3 and $f(\xi)=1-(\xi / 20)$. 


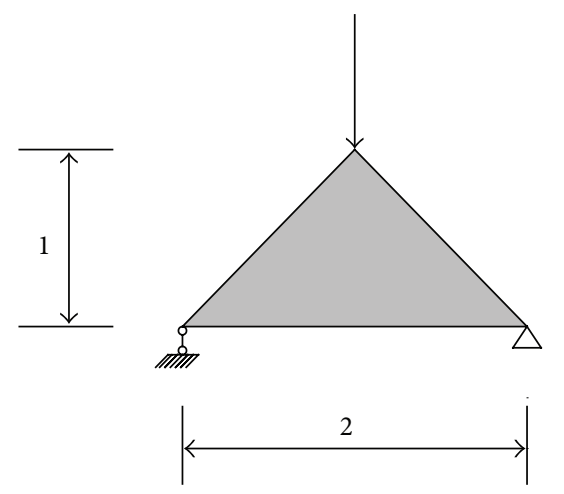

(a) FEM model

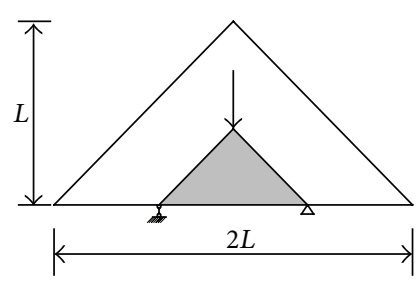

(b) Model 1

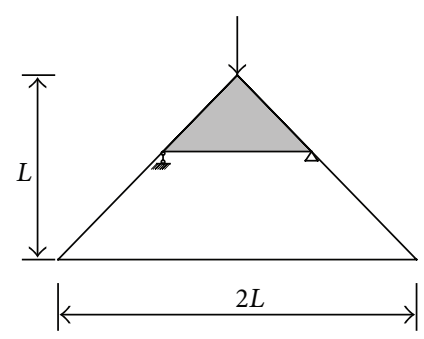

(c) Model 2

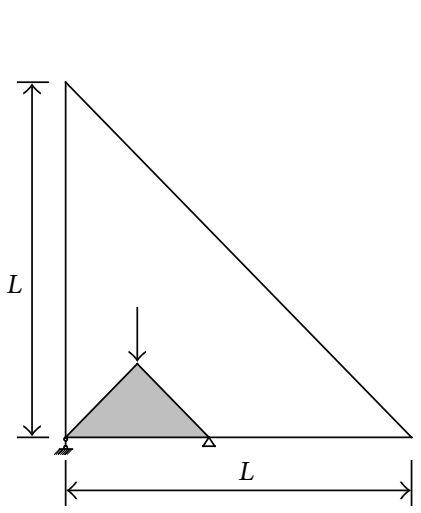

(d) Model 3

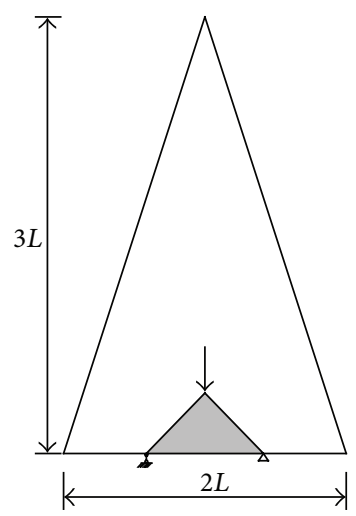

(e) Model 4

Figure 4: Models for test B (m).

TABLE 2: Material properties [17] for the cyclically loaded elements.

\begin{tabular}{lc}
\hline Parameter & Value \\
\hline$C_{1} C_{1}(\mathrm{MPa})$ & $2.067 \times 10^{7}$ \\
$C_{2}(\mathrm{MPa})$ & $6.545 \times 10^{6}$ \\
$C_{3}(\mathrm{MPa})$ & $2.138 \times 10^{6}$ \\
$C_{4}(\mathrm{MPa})$ & $6.890 \times 10^{5}$ \\
$C_{5}(\mathrm{MPa})$ & $2.103 \times 10^{5}$ \\
$C_{6}(\mathrm{MPa})$ & $4.49 \times 10^{4}$ \\
$C_{7}(\mathrm{MPa})$ & $6.95 \times 10^{3}$ \\
$E(\mathrm{MPa})$ & $1.15 \times 10^{5}$ \\
$\alpha_{1}$ & $3.077 \times 10^{7}$ \\
$\alpha_{2}$ & $5.961 \times 10^{6}$ \\
$\alpha_{3}$ & $1.172 \times 10^{6}$ \\
$\alpha_{4}$ & $2.024 \times 10^{5}$ \\
$\alpha_{5}$ & $2.851 \times 10^{4}$ \\
$\alpha_{6}$ & $2.276 \times 10^{3}$ \\
$\alpha_{7}$ & $4.140 \times 10^{2}$ \\
$v$ & 0.33 \\
\hline
\end{tabular}

The values of the intrinsic times, displacement, and residual strain of endochronic NMM are compared with the corresponding values of endochronic FEM in Table 4. It can be easily found that the displacements and the intrinsic times in models 1-4 are exactly the same as those in FEM. The computational results of the stress-strain by endochronic NMM and FEM are shown in Figure 6. The results indicate that the response of a physical cover in a mathematical cover will not be affected by the size, relative position, and shape of mathematical cover in endochronic NMM.

\section{Numerical Examples}

4.1. Simulation of Crack Problems with Endochronic NMM. This section is devoted to the demonstration of the capabilities of endochronic NMM in simulating the crack problems. A rectangular plate of $40 \mathrm{~m}$ wide and $40 \mathrm{~m}$ high with a piercing crack of $10 \mathrm{~m}$ in the centre is illustrated in Figure 7. Three different meshes, which are shown in Figure 8, have been considered. Tensions are applied on the top and bottom of the model with the maximum being equal to $12.8(\mathrm{MPa} \times$ $\mathrm{m})$. We adopt the same parameters for the endochronic model which are adopted in test A.

The distributions of von-Mises plastic strain with different DOFs are shown in Figure 9, which cannot be obtained by the original NMM. The distributions of von-Mises stress are also shown in Figure 9. It can be observed that the three results are almost the same despite minor discrepancies being found. It seems fair to claim that the endochronic NMM is capable of dealing with the elastoplasticity crack problems. 


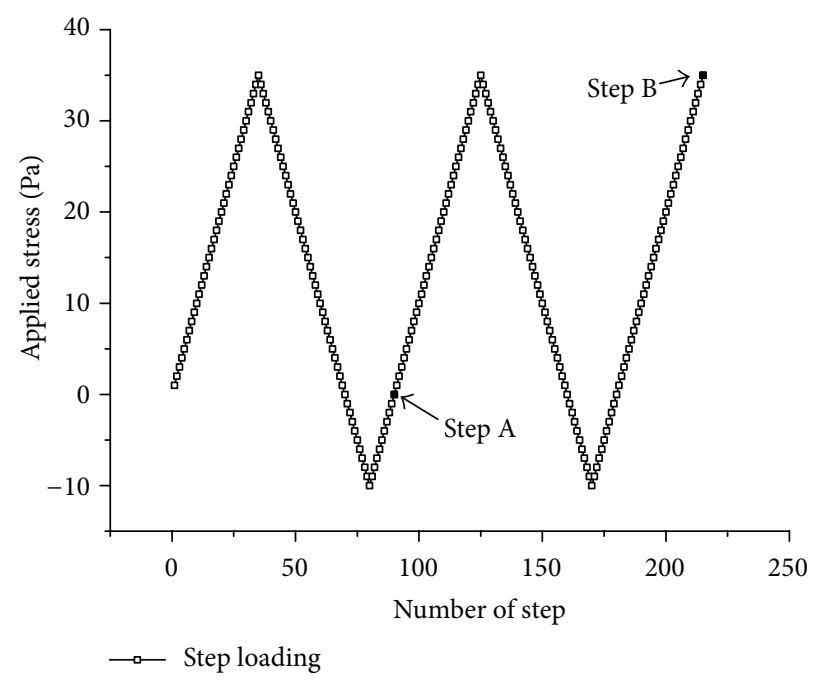

FIGURE 5: Cyclic loading diagram.

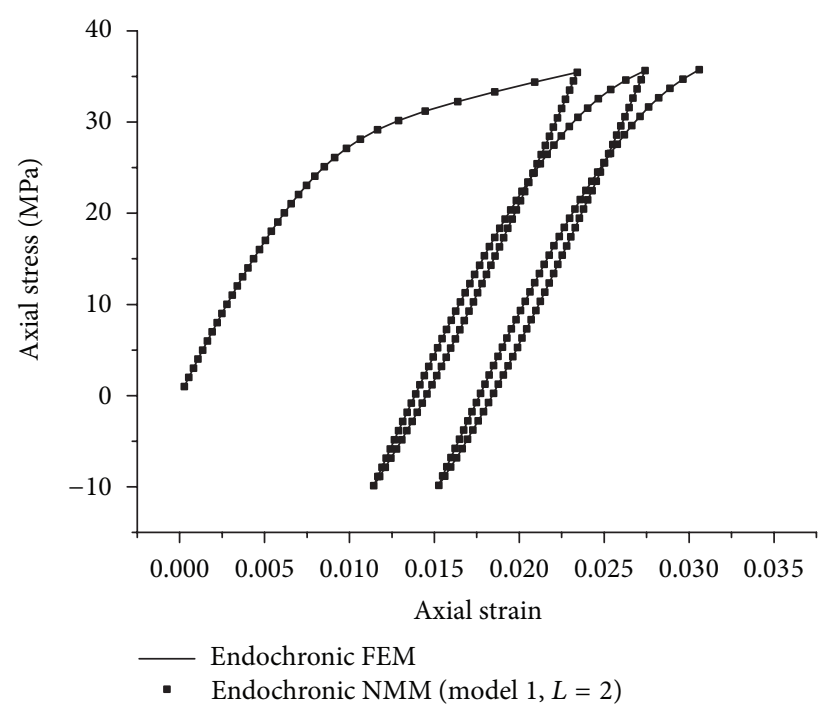

FIGURE 6: Computation results of axial stress and strain under cyclic loading.

TABLE 3: Material properties for test B.

\begin{tabular}{lc}
\hline Parameter & Value \\
\hline$C_{1} C_{1}(\mathrm{MPa})$ & 5000 \\
$C_{2}(\mathrm{MPa})$ & 200 \\
$E(\mathrm{MPa})$ & $1.15 \times 10^{5}$ \\
$\alpha_{1}$ & 500 \\
$\alpha_{2}$ & 15 \\
$v$ & 0.3 \\
\hline
\end{tabular}

4.2. Soft Clay Foundation under Traffic Loading Problem. In this section, a numerical investigation on the mechanical behavior of soft clay under the traffic moving loads is carried out to demonstrate the convenience of the endochronic
NMM. The geometry and mesh of the example are shown in Figure 10. Vehicle tire is modeled as a circle of radius $0.5 \mathrm{~m} . F$ is a concentrated force which is equal to $12.5 \mathrm{kN}$ and applied on the center of the tire. We let the tire move on the foundation five times with $1.00 \mathrm{~m} / \mathrm{s}$. In vertical direction, there is one observation point for every $0.5 \mathrm{~m}$. Figure 12 is the stress-strain relations of soil in a particular place under cyclic loading which is tested by Peng et al. [19]. We use the parameters of endochronic model which are listed in Table 5 and $f(\xi)=1-0.65 e^{-2.1 \xi}$. Figure 11 is the comparison of the results from numerical and test. To study the disturbing force generated by the tire, effect of the pore water pressure will be ignored.

Figure 12 shows the displacement of point E generated by endochronic NMM and conventional NMM. It could 


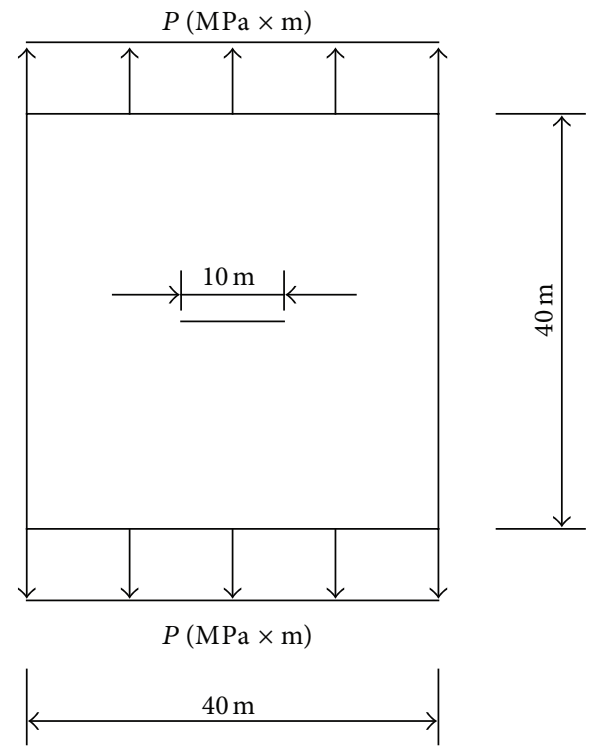

FIgURE 7: Geometric figure for crack problem.

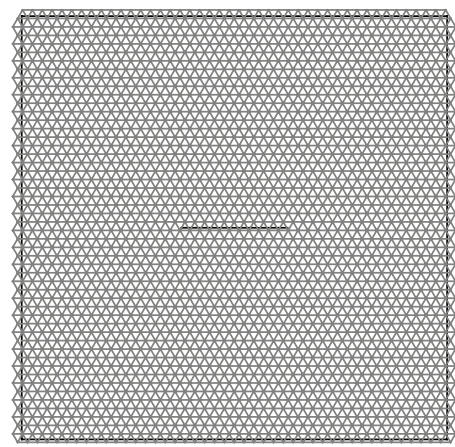

(a) $\mathrm{DOF}=4662$

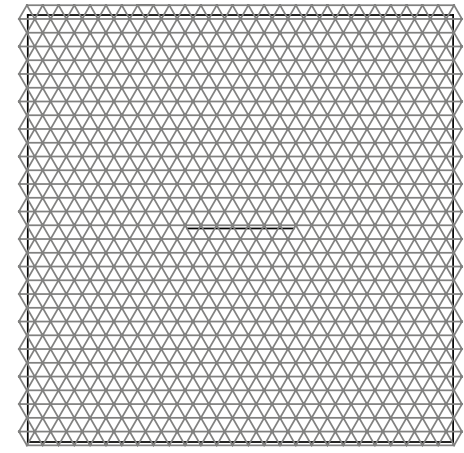

(b) $\mathrm{DOF}=1956$

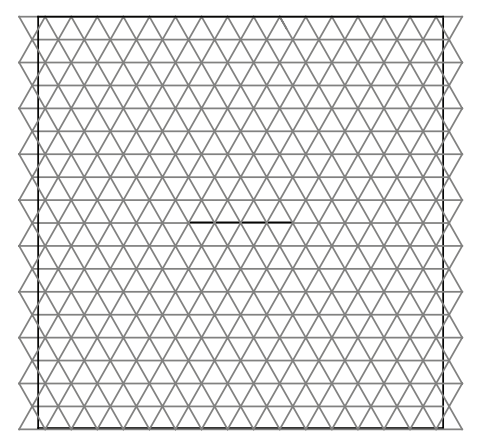

(c) $\mathrm{DOF}=706$

FIGURE 8: Three different meshes for crack problem.

TABLE 4: Aggregate of computational results.

\begin{tabular}{|c|c|c|c|c|}
\hline & Intrinsic time at step A & Residual strain at step A & Intrinsic time at step B & Displacement at step B \\
\hline Endochronic FEM & 0.022082 & 0.013895 & 0.039685 & 0.031449 \\
\hline \multicolumn{5}{|l|}{ Model 1} \\
\hline Endochronic NMM $(L=2)$ & 0.022082 & 0.013895 & 0.039685 & 0.031449 \\
\hline Endochronic NMM $(L=20)$ & 0.022082 & 0.013895 & 0.039685 & 0.031449 \\
\hline \multicolumn{5}{|l|}{ Model 2} \\
\hline Endochronic NMM $(L=2)$ & 0.022082 & 0.013895 & 0.039685 & 0.031449 \\
\hline Endochronic NMM $(L=20)$ & 0.022082 & 0.013895 & 0.039685 & 0.031449 \\
\hline \multicolumn{5}{|l|}{ Model 3} \\
\hline Endochronic NMM $(L=2)$ & 0.022082 & 0.013895 & 0.039685 & 0.031449 \\
\hline Endochronic NMM $(L=20)$ & 0.022082 & 0.013895 & 0.039685 & 0.031449 \\
\hline \multicolumn{5}{|l|}{ Model 4} \\
\hline Endochronic NMM $(L=2)$ & 0.022082 & 0.013895 & 0.039685 & 0.031449 \\
\hline Endochronic NMM $(L=20)$ & 0.022082 & 0.013895 & 0.039685 & 0.031449 \\
\hline
\end{tabular}



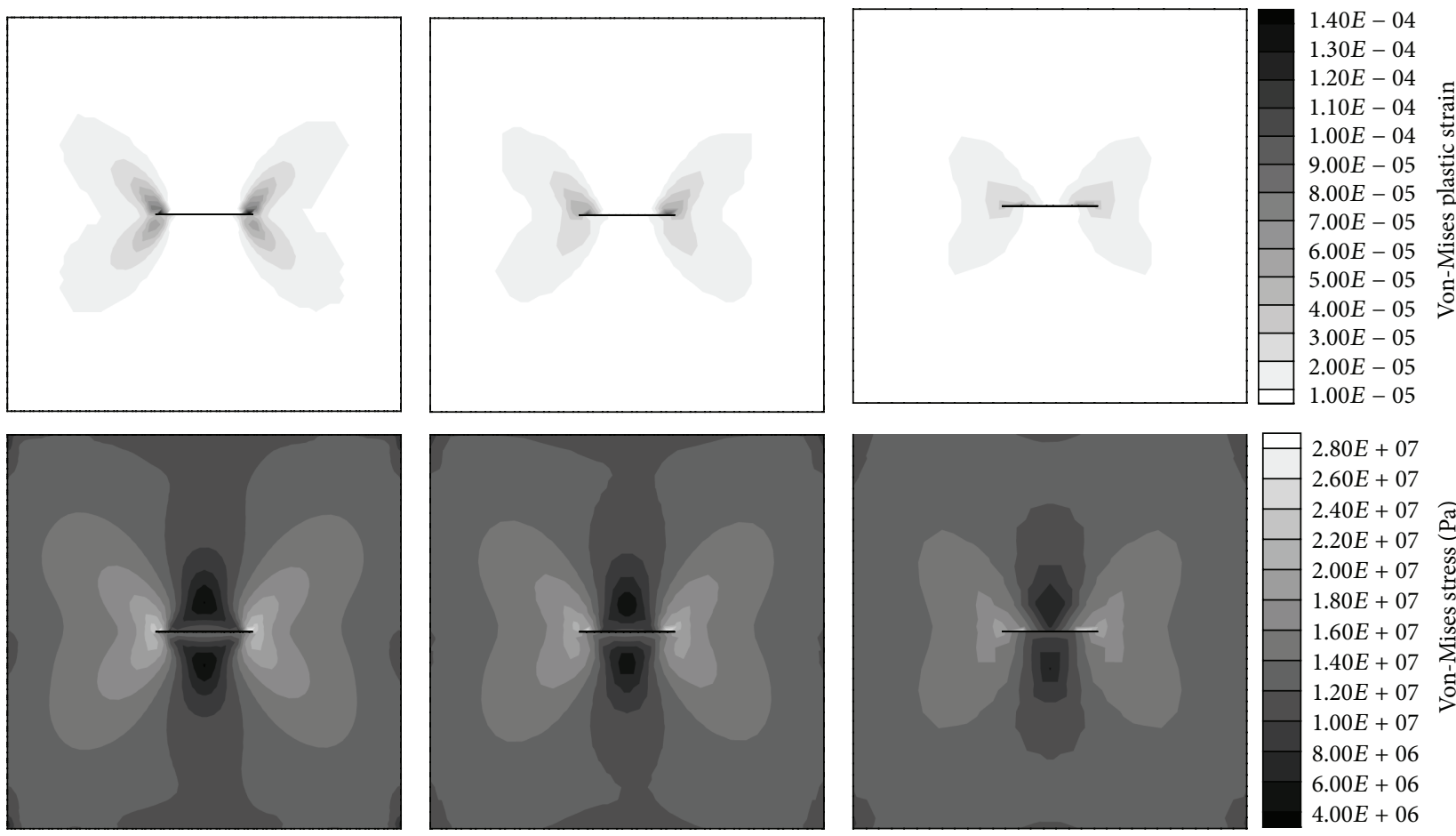

(a) $\mathrm{DOF}=4662$

(b) $\mathrm{DOF}=1956$

(c) $\mathrm{DOF}=706$

FIGURE 9: Distributions of von-Mises stress and von-Mises plastic strain with different DOFs.

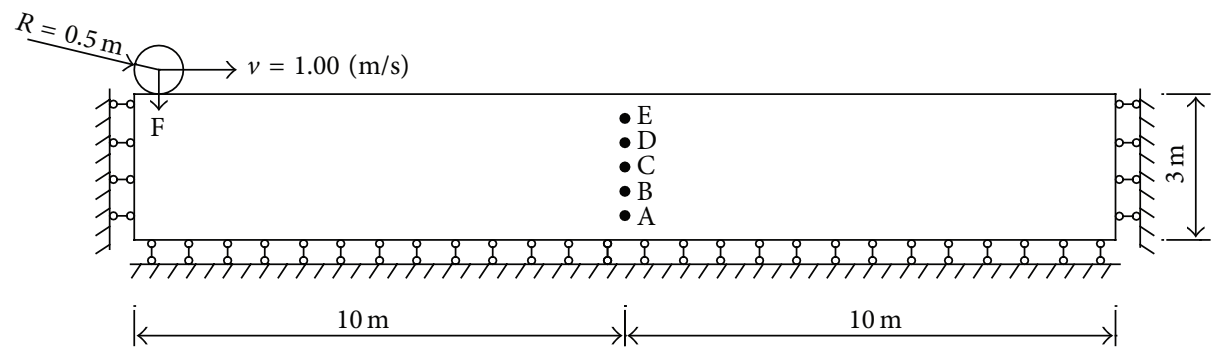

(a) Geometry and boundary conditions of the example

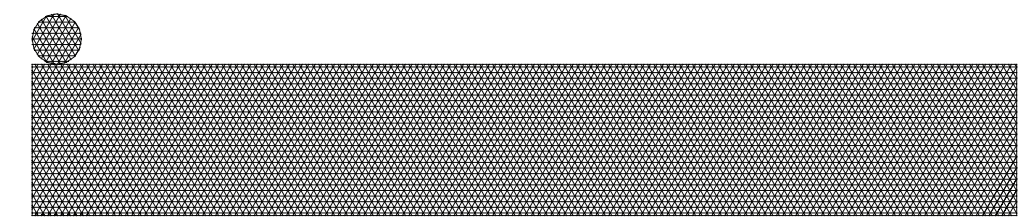

(b) Mesh of the example

FIGURE 10: Mesh and geometry of the example.

be observed that the endochronic NMM can calculate the land subsidence after the tire rolling through the foundation which cannot be simulated by conventional NMM. Figure 13 shows the additional stress generated by tire at points AE. It could be found that the curves of additional stress over time are similar to sine curves, which coincide with the conclusion by Hyodo and Yasuhara [20]. Figure 14 is the distribution of equivalent plastic strain when the tire passes the foundation for the first time. The results demonstrate that the endochronic NMM can deal with the elastoplasticity problems conveniently, especially at the problems which the mass movement and contact are in charge of.

\section{Conclusions}

The endochronic NMM, as an integration of the numerical manifold method and endochronic theory, is developed especially for elastoplasticity problems. Endochronic theory, 


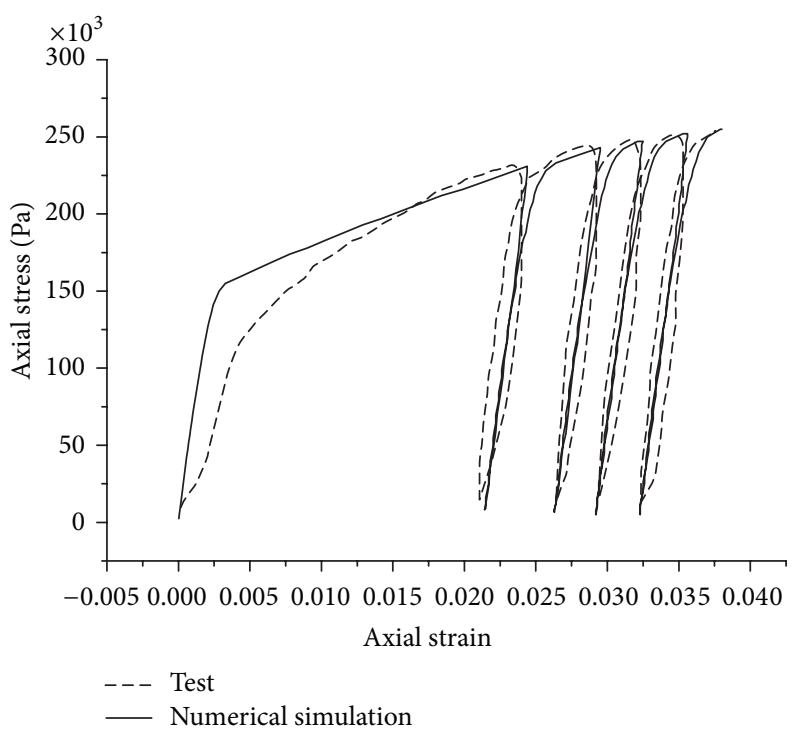

FIGURE 11: Stress-strain relations of soil.

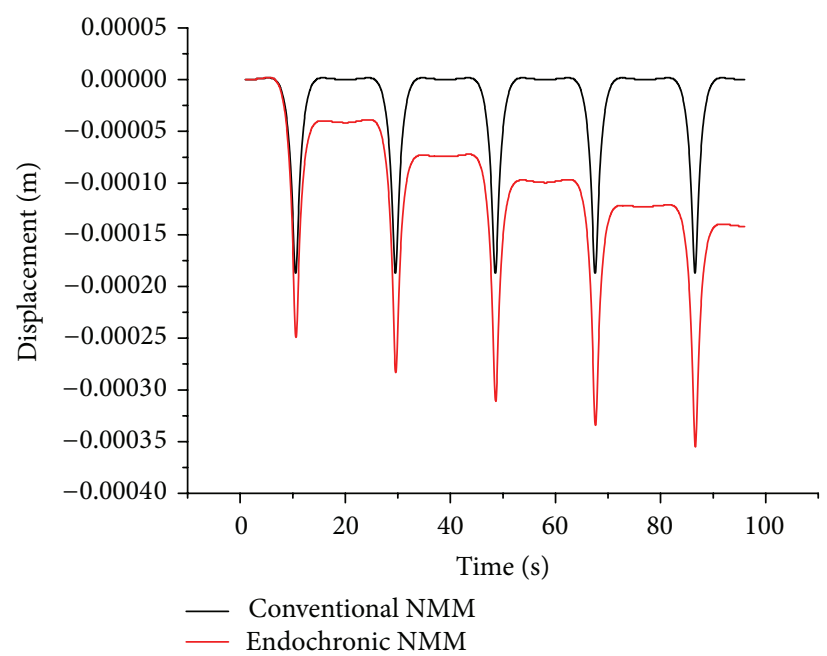

FIGURE 12: Displacement of point E.

TABLE 5: Parameters of endochronic model for soft clay foundation under traffic loading problem.

\begin{tabular}{lc}
\hline Parameter & Value \\
\hline$C_{1} C_{1}(\mathrm{~Pa})$ & $4.00 \times 10^{8}$ \\
$C_{2}(\mathrm{~Pa})$ & $1.20 \times 10^{8}$ \\
$E(\mathrm{~Pa})$ & $8 \times 10^{7}$ \\
$\alpha_{1}$ & $7.00 \times 10^{3}$ \\
$\alpha_{2}$ & $4.00 \times 10^{2}$ \\
$v$ & 0.3 \\
\hline
\end{tabular}

which is one of the most typical elastoplasticity theories for modeling material behavior, has been successfully introduced into the presented version of NMM in this paper. Results of tests show that the endochronic NMM is a very useful and accurate method for plasticity analysis. The numerical examples of crack problem and soft clay foundation under traffic loading problem demonstrate the convenience of the endochronic NMM in analyzing elastoplasticity discontinuous problems.

\section{Conflict of Interests}

The authors declare that there is no conflict of interests regarding the publication of this paper.

\section{Acknowledgments}

This research was supported by National Natural Science Foundation of China under Grant no. 51109028 and the State 


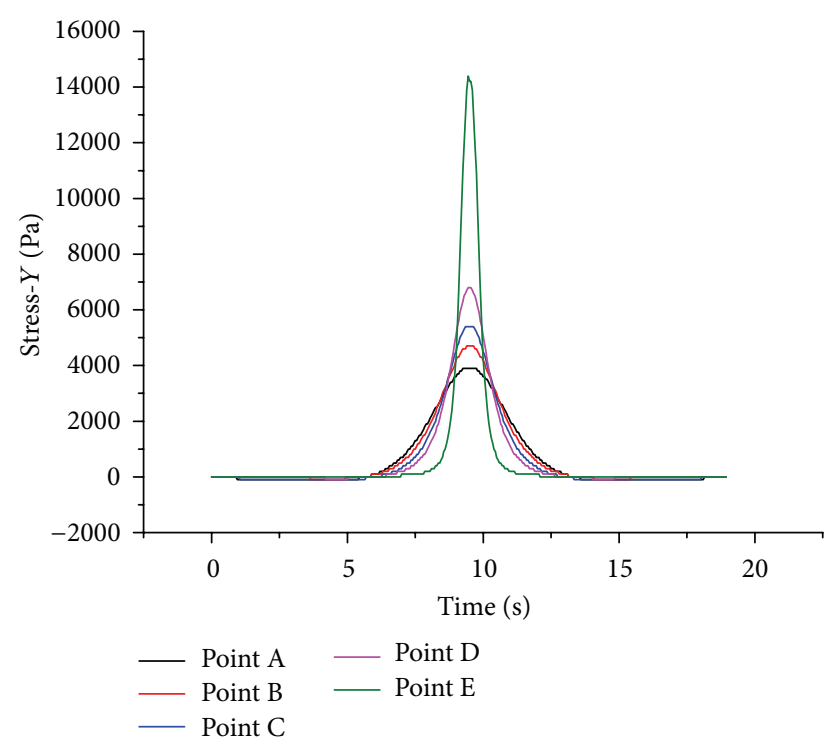

FIgURE 13: Additional stresses change over time for points A-E.

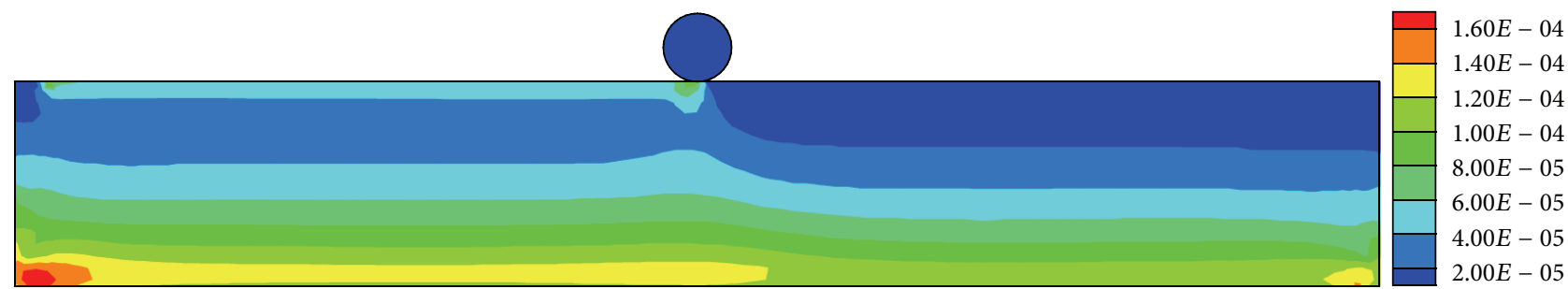

FIGURE 14: Distribution of equivalent plastic strain.

Scholarship Fund of China to pursue study in USA as a visiting scholar under Grant no. 201208210208.

\section{References}

[1] G. H. Shi, "Manifold method of material analysis," in Transactions of the 9th Army Conference on Applied Mathematics and Computing, pp. 57-76, US Army Research Office, Minneapolis, Minn, USA, 1991.

[2] G. H. Shi, "Numerical manifold method," in Proceedings of the 2nd International Conference on Analysis of Discontinuous Deformation, pp. 1-35, Kyoto, Japan, 1997.

[3] G. W. Ma, X. M. An, and L. He, "The numerical manifold method: a review," International Journal of Computational Methods, vol. 7, no. 1, pp. 1-32, 2010.

[4] S. Li, Y. Cheng, and Y.-F. Wu, "Numerical manifold method based on the method of weighted residuals," Computational Mechanics, vol. 35, no. 6, pp. 470-480, 2005.

[5] G. W. Ma, X. M. An, H. H. Zhang, and L. X. Li, "Modeling complex crack problems using the numerical manifold method," International Journal of Fracture, vol. 156, no. 1, pp. 21-35, 2009.

[6] H. F. Gao and Y. M. Cheng, "A complex variable meshless manifold method for fracture problems," International Journal of Computational Methods, vol. 7, no. 1, pp. 55-81, 2010.

[7] H. H. Zhang, L. X. Li, X. M. An, and G. W. Ma, "Numerical analysis of 2-D crack propagation problems using the numerical manifold method," Engineering Analysis with Boundary Elements, vol. 34, no. 1, pp. 41-50, 2010.

[8] K. Terada and M. Kurumatani, "Performance assessment of generalized elements in the finite cover method," Finite Elements in Analysis and Design, vol. 41, no. 2, pp. 111-132, 2004.

[9] S. C. Li and Y. M. Cheng, "Meshless numerical manifold method based on unit partition," Acta Mechanica Sinica, vol. 36, no. 4, pp. 496-500, 2004.

[10] S. C. Li and Y. M. Cheng, "Enriched meshless manifold method for two-dimensional crack modeling," Theoretical and Applied Fracture Mechanics, vol. 44, no. 3, pp. 234-248, 2005.

[11] H. W. Zhang and L. Zhou, "Numerical manifold method for dynamic nonlinear analysis of saturated porous media," International Journal for Numerical and Analytical Methods in Geomechanics, vol. 30, no. 9, pp. 927-951, 2006.

[12] H. F. Gao and Y. M. Cheng, "Complex variable numerical manifold method for elasticity," Acta Mechanica Sinica, vol. 41, no. 4, pp. 480-488, 2009.

[13] K. C. Valanis, "A theory of viscoplasticity without a yield surface-part I. General theory," Archives of Mechanics, vol. 23, no. 4, pp. 517-534, 1971.

[14] K. C. Valanis, "A theory of viscoplasticity without a yield surface-part II. Application to mechanical behavior of metals," Archives of Mechanics, vol. 23, pp. 535-551, 1971.

[15] K. C. Valanis and J. Fan, "Endochronic analysis of cyclic elastoplastic strain fields in a notched plate," Journal of Applied Mechanics, vol. 50, no. 4, pp. 789-794, 1983. 
[16] K. C. Valanis and H. E. Read, "An endochronic plasticity theory for concrete," Mechanics of Materials, vol. 5, no. 3, pp. 277-295, 1986.

[17] C. F. Lee, "Recent finite element applications of the incremental endochronic plasticity," International Journal of Plasticity, vol. 11, no. 7, pp. 843-865, 1995.

[18] H. S. Lamba and O. M. Sidebottom, "Cyclic plasticity of nonproportional paths-part 1. Cyclic hardening, erasure of memory, and subsequent strain hardening experiments," Journal of Engineering Materials and Technology, vol. 100, no. 1, pp. 96-103, 1978.

[19] L.-Y. Peng, T. Li, and J.-K. Liu, "Deformation property of unsaturated compacted silts under cyclic loading," Journal of Beijing University of Technology, vol. 36, no. 11, pp. 1504-1509, 2010.

[20] M. Hyodo and K. Yasuhara, "Analytical procedure for evaluating pore-water pressure and deformation of saturated clay ground subjected to traffic loads," in Proceedings of the 6th International Conference on Numerical Methods in Geomechanics, pp. 653-658, Rotterdam, The Netherlands, 1988. 


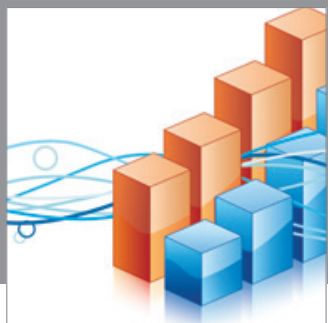

Advances in

Operations Research

mansans

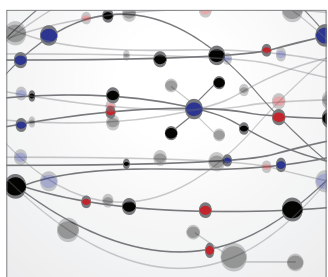

The Scientific World Journal
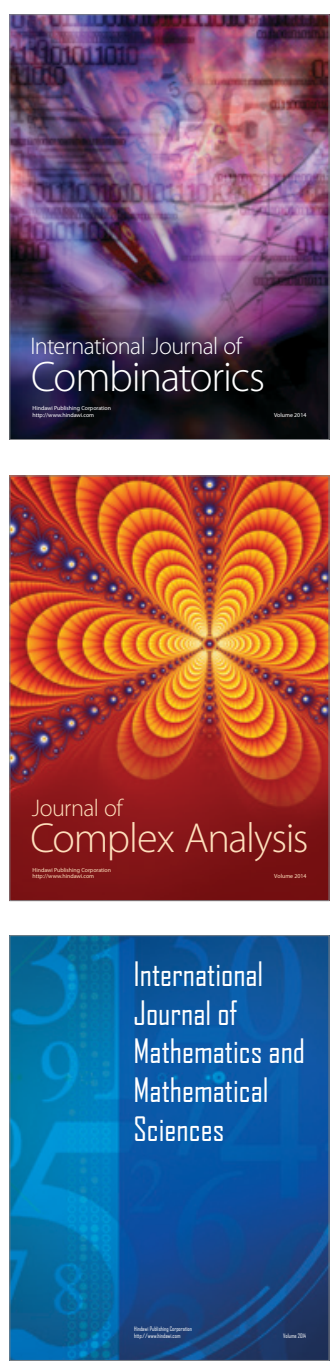
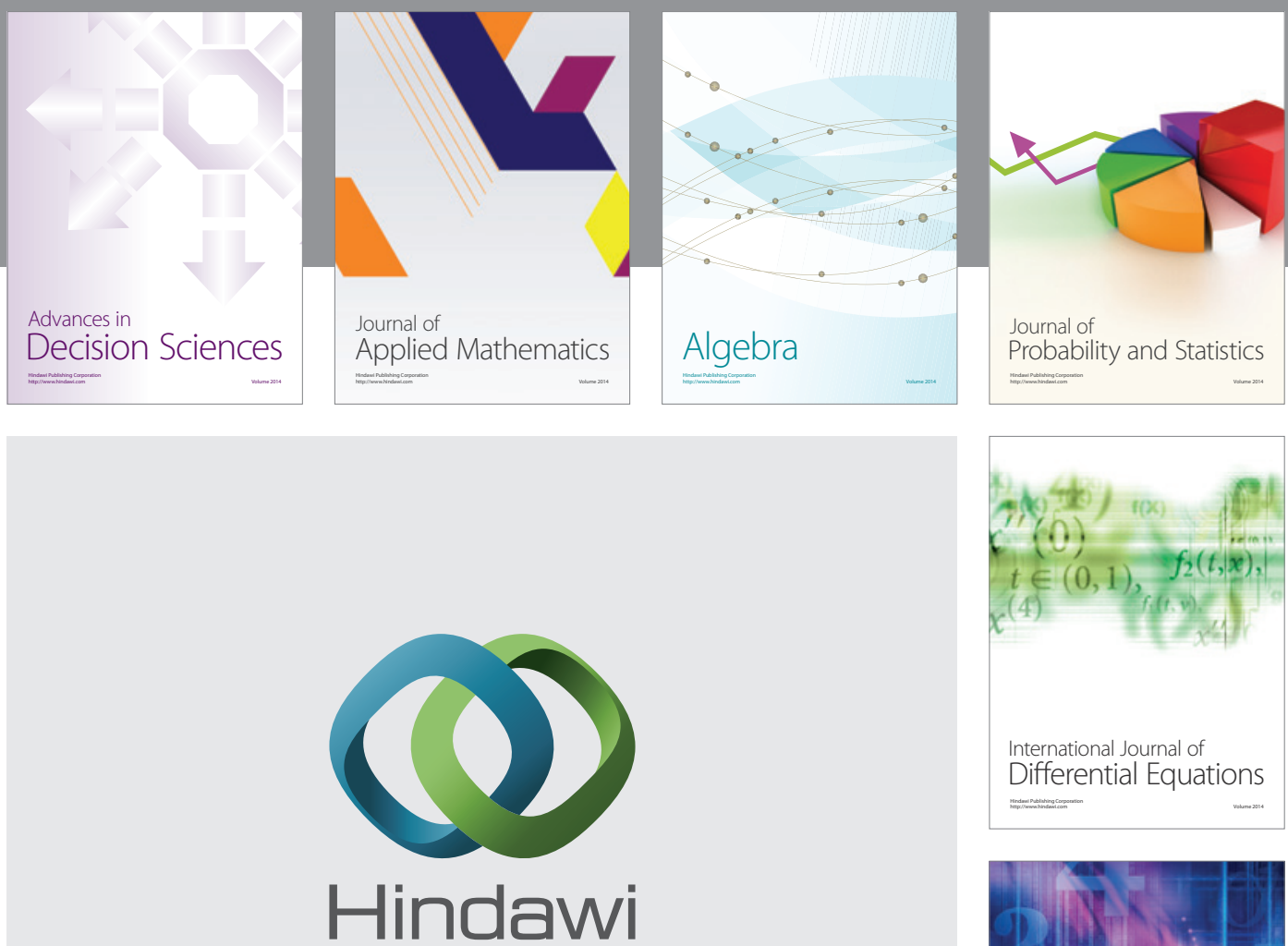

Submit your manuscripts at http://www.hindawi.com
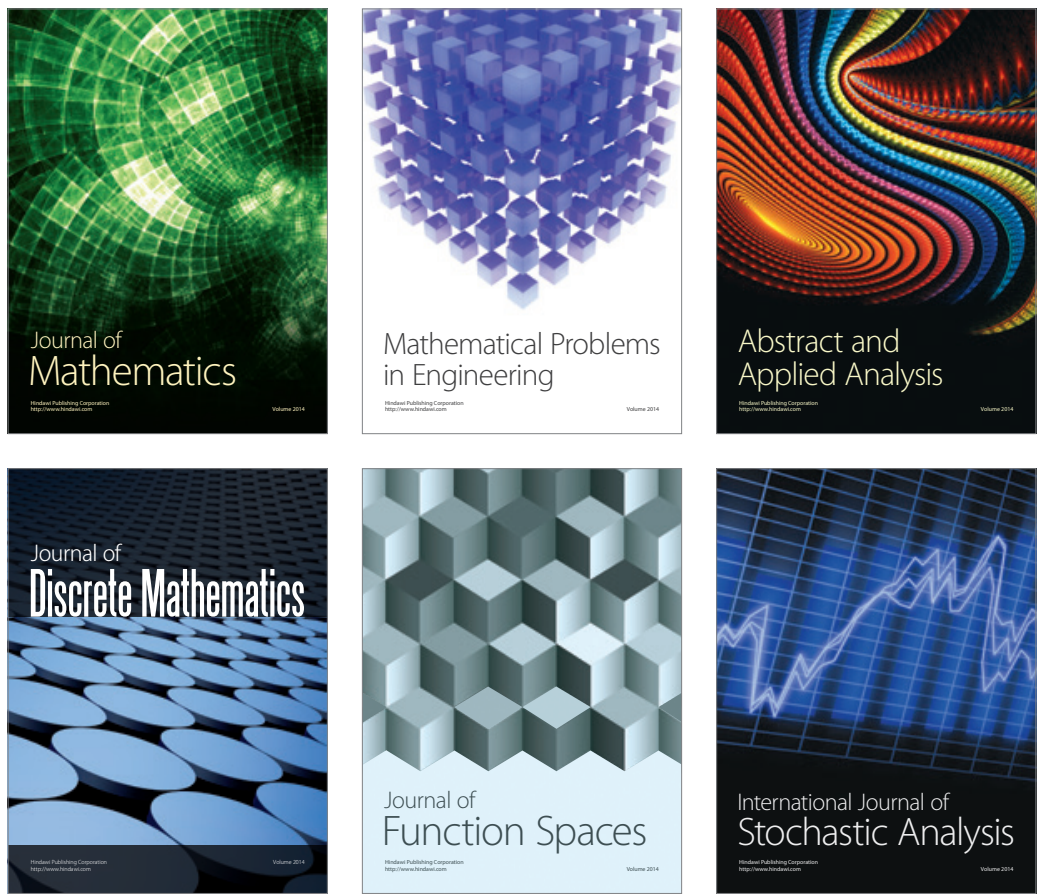

Journal of

Function Spaces

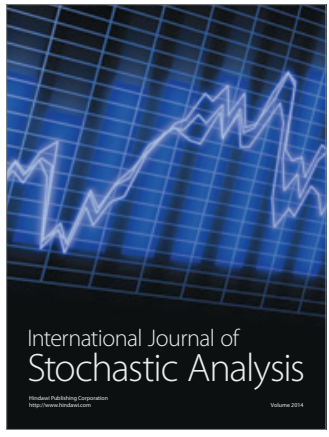

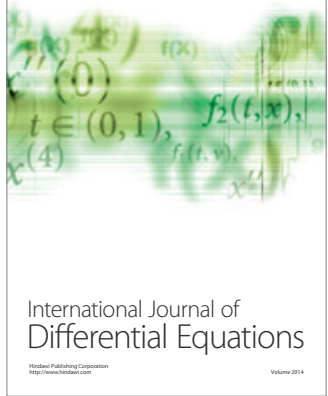
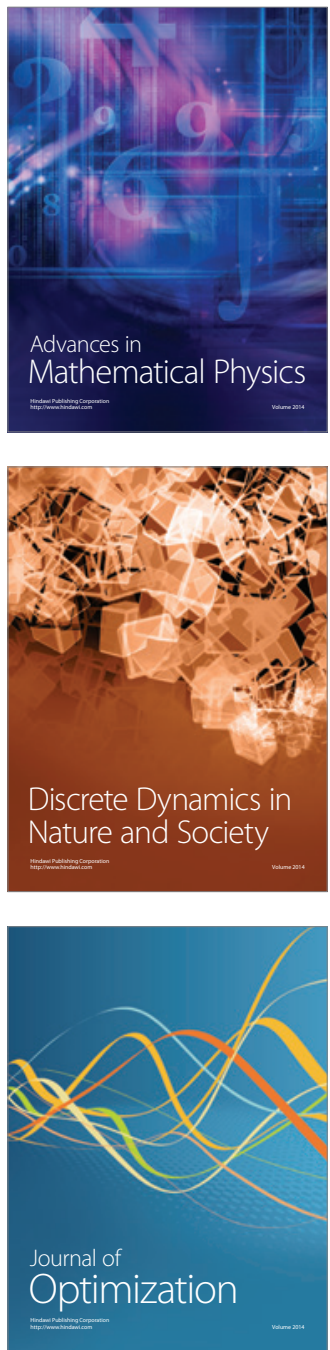